Research Article

\title{
Application and Research of the Intelligent Management System Based on Internet of Things Technology in the Era of Big Data
}

\author{
Xiaojing $\mathrm{Lv}^{1}$ and Minghai $\mathrm{Li} \mathbb{D}^{2}$ \\ ${ }^{1}$ Gradutate School of Management of Technology, Pukyong National University, Busan 48547, Republic of Korea \\ ${ }^{2} X i$ 'an University of Architecture \& Technology, Xi'an 710055, Shaanxi, China \\ Correspondence should be addressed to Minghai Li; jdjskfgs@xauat.edu.cn
}

Received 14 May 2021; Revised 1 June 2021; Accepted 4 June 2021; Published 16 June 2021

Academic Editor: Sang-Bing Tsai

Copyright (c) 2021 Xiaojing Lv and Minghai Li. This is an open access article distributed under the Creative Commons Attribution License, which permits unrestricted use, distribution, and reproduction in any medium, provided the original work is properly cited.

\begin{abstract}
With the in-depth application of the Internet of Things, many emerging technologies are changing the global industry landscape on an unprecedented scale. At the same time, they also provide an opportunity for the development of intelligent management to break through the bottleneck. The proposal and evolution of the concept of intelligent management makes the development of management technology more advanced. Therefore, the introduction of the Internet of Things technology into intelligent management has very important research significance and value. First, conduct research on the origin and current situation of the Internet of Things technology at home and abroad and understand the relevant theories and cutting-edge technologies of the Internet of Things technology. Second, take the enterprise as an example to study the process flow and existing problems in the logistics link in the factory, combine the management concept of the Internet of Things, and integrate IC card identification technology, RFID radio frequency identification technology, barriers and ground sensing technology, and OPC/PLC. Third, combined with PLC/OPC technology, the design and integration of the software system and hardware system are realized. The experimental results show that the system modules have been tested to provide company information management, employee multifactor predictive analysis, and efficient batch efficiency evaluation, which has a certain value for company data management and data analysis and mining, and improve company efficiency by more than $30 \%$.
\end{abstract}

\section{Introduction}

With the application of science and technology and the widespread use of the Internet, we have now entered the era of digital information explosion, which is the "big data era." The combination of management ideas and methods with artificial intelligence technology and intelligent management is an important part of management practice.

The intelligent management system can use information communication technology and machine intelligence to achieve the automatic and intelligent requirements of all management behaviors through information management and intelligent system platforms. Depersonalized intelligent management has brought about a revolution in management science without any problem and has become an important subject of management practice. In addition, the smart heating system that is common in smart management systems can not only improve the efficiency and benefits of heating management but also promote energy conservation, emission reduction, and environmental protection.

With the rapid development of big data, the Internet of Things, cloud computing, and logistics have gradually become an important part of promoting social and economic development and are constantly striding forward to a brandnew intelligent information visualization brand. Aiming at the current economic and social development trend, Yi has established an intelligent logistics information management model based on the Internet of Things and cloud computing through the analysis of the logistics industry, which can provide intelligent users with different privileges and query logistics information according to the needs of system users [1]. The latest growth and expansion in the Internet of 
Things (IoT) field provides a huge business prospect towards a new era of smart cities. The insight of smart cities is widely favored because it improves the living standards of citizens by connecting smart transportation, smart parking, smart environment, medical, and other regulations. Babar and Arif proposed city architecture based on big data analysis. However, the complexity of this framework is too high, leading to errors in the analysis process [2]. The Internet of Things (IoT) is an emerging technology in the 21st century. It was described by Mohamed and Zulhuda as the first real development of the Internet that can have a positive or negative impact on all aspects of life. However, IoT, like other technologies, has disadvantages, especially in terms of data privacy and security [3].

Based on a full understanding of the actual conditions of production and operation in the heating industry, this study introduces the concept of intelligent management and proposes a solution for intelligent management of heating metering. Intelligent management is to automate management decisions and engineer management behaviors. Information management and information systems are undoubtedly the theoretical and technical foundations of intelligent management. The heat metering intelligent management platform promotes the rational consumption of heat energy to achieve energy conservation, environmental protection, fair trade, and best use of materials. The main research results of this study include the following five points.

(1) Put forward the concept of intelligent management and elaborated the intelligent management solution that combines modern information technology and Internet of Things technology in heating management

(2) From the perspective of technical realization, an intelligent management platform for heating metering was analyzed and planned

(3) According to the difference between the new and old buildings and the structure, the intelligent management platform can mix the temperature measurement program and the on-off method measurement program

(4) According to the current situation of the heating industry, select and design a suitable heat meter and installation plan

(5) Integrate the concept of Internet of Things, cloud computing, and "Internet + " to monitor the whole process of heating services and provide prediction and decision-making basis through big data mining

\section{Application of the Intelligent Management System}

2.1. Big Data. The concept of big data originally referred to a very large amount of data [3]. In the early days, its amount has exceeded the range of conventional computer calculations. Over time, the term in the IT industry is a collection of large amounts of data, which means that conventional software tools cannot capture these data within a specific time frame $[4,5]$. Management and processing records are huge, complex, and diverse information, and new processing models are needed to have stronger decision-making capabilities [4].

2.2. Internet of Things. The Internet of Things is an important development of modern information technology. As the name suggests, the Internet of Things is still a part of the Internet and an extension of the Internet. Its core and foundation are still the Internet [6]. The Internet of Things is the Internet in real life, that is, a network that connects objects to work and life through identification technology, identification technology, and computer technology. The connection of the six basic technologies of the Internet of Things is usually used in the concept of "Internet +," thereby linking network technologies, cloud computing, M2M, extensive computing, RFID, and sensor technologies $[7,8]$.

2.3. Intelligent Management System. First, relying on the Internet of Things technology, we propose an intelligent management solution for heating metering, build a corresponding intelligent management system platform, which can realize remote real-time reading of heating meter data, and can achieve 24/7 uninterrupted management of data to ensure data accuracy and real-time $[9,10]$. The second is to study the technical solutions for heat metering, build a heat metering intelligent management platform based on this model, put forward a set of intelligent heat metering solutions based on the Internet, and design the real-time guarantee and management realization of the heat metering intelligent management platform, as well as the engineering case analysis, as shown in Figure 1.

The Internet of Things network layer is responsible for the transmission of network data, including the access module part and the Internet of Things access network module [11]. The Internet of Things module has a unique terminal for each Internet of Things and an Internet of Things gateway for data collection. IoT gateways can define different gateways according to different scenarios, such as industrial, home, and shared gateways [12]. There are two types of access network modules: wired or wireless. It is responsible for preserving information in the physical world and fully understanding external information [13]. The perception level mainly includes the collaborative information processing level, the data acquisition level, and the short-distance communication protocol.

The network initialization assigns small random numbers to the weight vectors of the output layer and normalizes them to obtain $w(j=1,2, \ldots, m)$ and establish the initial winning field $N(0)$ and the initial learning rate $\eta$ value. $m$ is the number of neurons in the output layer.

$$
\frac{1}{2} \eta=\sqrt{N+\left(\frac{w_{i}(a)}{m}\right)} .
$$

An input pattern is randomly selected from the training set and normalized to obtain $X(i=1,2, \ldots, n)$, where $n$ is the number of neurons in the input layer. 


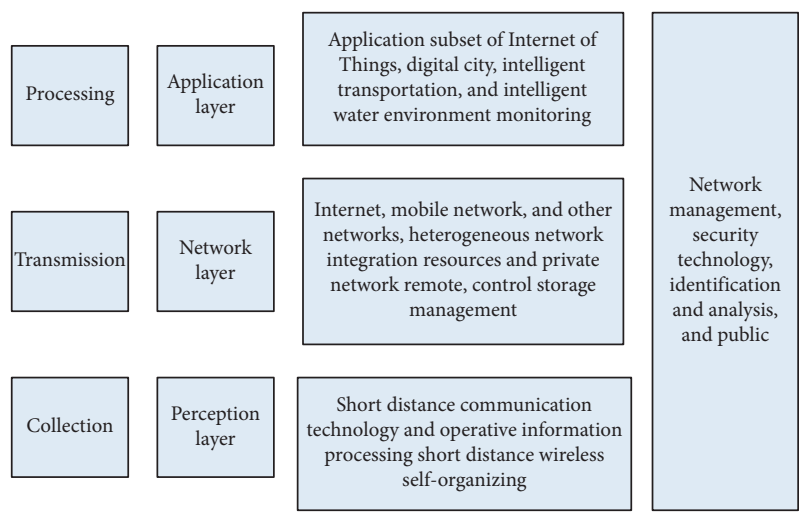

FIGURE 1: The architecture of the internet of things.

$$
\eta=N_{j}\left[x_{m}-w_{i j}(t)\right]
$$

Calculate the dot product of $X$ and $w$ and find the winning node $j$ with the largest dot product. If the input mode is not normalized, the winning node with the smallest distance should be found from it.

$$
d_{j}=\left\|X_{t}-W_{j}\right\|=\sqrt{\sum_{j=1}^{m}}\left[X_{i}-W_{j}\right]^{2} .
$$

Let $j$ be the center to determine the weight adjustment domain at time $t$. Generally, the initial domain $N(0)$ is large, and $N(t)$ shrinks with the training time during the training process.

$$
j \in N_{j}^{*}(t) \text {. }
$$

Adjust the weights of all nodes in the winning field $N(t)$,

$$
w_{i j}(t+1)=w_{i j}(t)+a(t, N)
$$

Before introducing structural risk minimization, first introduce the following concept: suppose VC-dimension $h$ is finite, then the following formula holds with probability $\geq 1-\eta$ :

$$
R(a) \leq R_{\mathrm{emp}}(a)+\forall a \in \wedge,
$$

where $\eta$ is a small integer,

$$
\varepsilon=4\left[\left(\ln \left(2 \int / h\right)+1\right)-\ln (\ln (\eta / 4)) \int\right] .
$$

For the linearly separable training set, its samples all meet the following condition:

$$
y_{i}\left(\left(w \cdot x_{i}\right)+b\right)-1 \geq 0 \text {. }
$$

Among them, the data collection plane is composed of various sensors and receives information such as time information and status information in the real world through various sensors [14].

\section{Design of the Intelligent Management System for Heating Metering}

3.1. Planning and Construction of Heat Metering Intelligent Management Mode. The heating unit is the main body of responsibility in the heat supply measurement management and the main body of the heat supply measurement management $[15,16]$. Heat metering is used in the environment of district heating or central heating, in order to meet demand, and at the same time to strengthen the energy-saving and environmental protection awareness of heating users in the process of use, and to protect the interests of both the heating unit and the heating user, the heating service users are charged according to the amount of each household [17]. The heating metering is for the units and individuals that centrally provide heating in cities and towns. Use heat to measure and measure the heat supplied by the heat source of the heating system [18]. There are mainly the following five common heating measurement methods: heat distribution measurement method, on-off time area method, flow temperature method, temperature area method, and household heat meter method, as given in Table 1.

\subsection{Heating Metering Intelligent Management System Should} Be Designed. The heating metering intelligent management system is under the Internet of Things. It can manage and control the centralized heating at the same time [19]. It is a comprehensive intelligent management system. First of all, the heating intelligent management platform can realize the unity of the entire pipe network and even the user management, covering the entire heating system for effective supervision, management and control, and second, it can realize the operation of the complete heating system and the fine control of the operation process of the entire system, which can successfully save the heating unit's expenditure on system management [20], time, and energy, and further achieve the goal of energy-saving control in all aspects of the heating system.

The intelligent management system integrates the needs of all aspects and regards saving costs, saving energy, and reducing overall consumption as the main goals of the design. It includes different parts such as the heat meter data statistics system, heat control system, and temperature adjustment system, which are tailor-made for heating units [21]. It is to complete remote centralized statistical analysis of heat metering, temperature control valve status, and room temperature information, thereby achieving the goal of energy saving and environmental protection to a large extent [22]. The intelligent heat management platform is the cornerstone of the management and monitoring of the entire city heating network. The platform uses GIS technology and selective heating models for integrated development and makes full use of these two technologies to obtain information about pipeline network resources. The purpose of integration is to store, manage, and analyze changes in water temperature, water volume, pressure, and other conditions [23]. The management and management of the entire heating system process have carried out in the heating pipe network. The time management and temperature zone management system of the intelligent heating metering management platform and the central computer control system form a cooperative organization, which is very suitable for different areas under a unified heat source. 
TABLE 1: The table of heat metering mode selection.

\begin{tabular}{|c|c|c|c|}
\hline $\begin{array}{l}\text { The end of the } \\
\text { building }\end{array}$ & \multicolumn{2}{|c|}{ In building system } & Applicable methods \\
\hline \multirow{3}{*}{ Radiator } & \multicolumn{2}{|c|}{$\begin{array}{l}\text { Household independence } \\
\text { level }\end{array}$} & $\begin{array}{l}\text { Heat distribution metering method, household heat meter metering method, and on-off } \\
\text { time area method }\end{array}$ \\
\hline & \multirow{2}{*}{$\begin{array}{c}\text { Vertical } \\
\text { loop }\end{array}$} & $\begin{array}{l}\text { Double } \\
\text { tube }\end{array}$ & Heat distribution metrology \\
\hline & & Single tube & Heat distribution measurement and flow temperature method \\
\hline Floor heating & \multicolumn{2}{|c|}{$\begin{array}{l}\text { Household independence } \\
\text { level }\end{array}$} & Household heat meter measurement method and on-off time area method \\
\hline Fan coil unit & \multicolumn{2}{|c|}{$\begin{array}{l}\text { Household independence } \\
\text { level }\end{array}$} & Measurement method of household heat meter \\
\hline
\end{tabular}

The heating is integrated and coordinated and controlled. It is known that each heating pipe network and each heating device provide different heat consumption at different times, and the unit of heat consumption varies with time and area. Especially for those who use different heat at different times of the day, provide different temperatures at different times for special start-up control, and for certain units such as schools and office buildings to provide differential heating at different times such as day and night, weekend, and workdays, it is very suitable. The heat metering intelligent management platform solves the problem of heating energy waste caused by the use of the same temperature to heat different units at different periods of time, which is common in most traditional heating systems and realizes the transition from traditional heating to "on-demand heating" "The change [24]." The frame diagram is shown in Figure 2:

The overall design of this platform can be roughly divided into four layers: data acquisition layer, wireless network transmission layer, data processing layer, and management system layer. First, the data collection layer is the IoT perception layer of the entire platform, which transmits the information collected by the transmitter to the collector through the bus. Then, the heating meter data on the collector is transmitted to the platform through the GPRS network, which is the Internet of Things network layer of the entire platform. The GPRS network is the connection bridge between the platform's data collection layer and the data processing layer and is the guarantee of smooth upper and lower two-way communication. The third is the data processing layer of the platform, which is the application layer in the Internet of Things. The data collected by the collector are sent to the intelligent management platform of the data processing layer through the GPRS wireless network to realize the management of the data. Through the mastery of these data, not only can we understand the user's heat use data but also can analyze these data and know the user's heat use habits and other in-depth information, which is convenient for heat supply unit management and flexible heating.

\section{Experimental Analysis}

Big data is an unimaginable and abstract collection of huge data, but its processing process will also appear routine with the cooperation of emerging technologies. Its processing

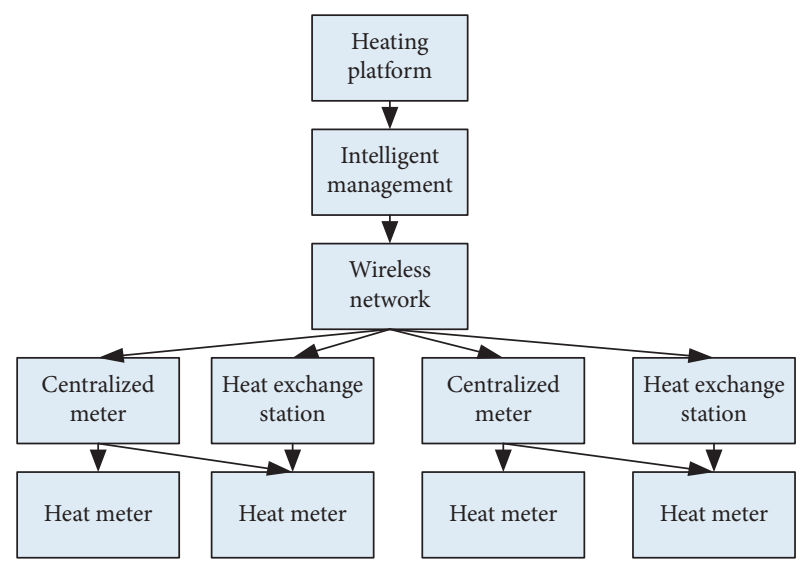

FIGURE 2: The master-plan organization chart of the system.

process is mainly divided into data collection, data processing, and data storage. As a result, the big data technology is now rapidly developing, and the heating industry is facing reorganization. In the process of heating system reform, effectively mining the value of data is the key to the success of the reform. In the context of the development of big data, the effective realization of the value of data is a prerequisite for improving the sound operation of heating companies and a guarantee for improving corporate performance. Many of the government's regulatory actions are now realized using data. In addition, companies can use the collected data to improve services and increase user satisfaction. Make good use of big data technology to integrate and utilize data resources, discover the problems behind it through data, predict problems in advance, respond quickly, save resources, and accelerate the popularization and utilization of big data technology. However, people have to consider its restrictive effects while enjoying the convenience brought by big data. The biggest constraint is that people's privacy is threatened. "Notice to permission" has become the consensus basis for the implementation of privacy policies around the world. But there are still problems. Apart from this, "anonymization" is not a way to solve the privacy problem. As more and more data are collected by a certain subject, we can instead pass other data, according to a more accurate guess of the subject's private information. In the same way, the fuzzification of certain data cannot protect personal privacy because other data information will 


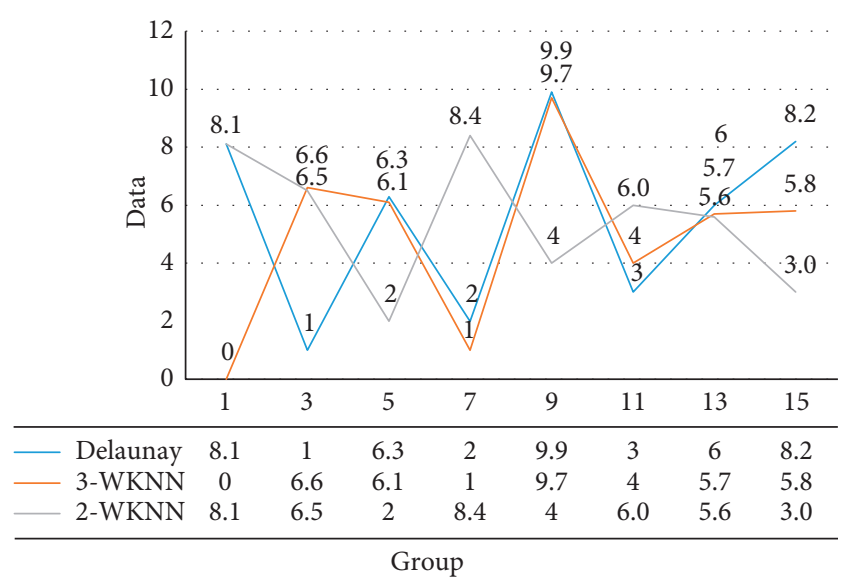

Figure 3: Comparison of matching algorithm positioning error.

eventually be calculated into obscure data information. In summary, in the era of big data, no matter whether it is "information and permission," "anonymization," or "fuzzification," the privacy of the subject cannot be effectively protected. The privacy threats brought by big data are involved in various fields and also restricting the development of daily necessities design.

The experimental result of positioning the moving target using the positioning algorithm based on Delaunay triangulation is shown in Figure 3. It can be seen from the experimental results that the algorithm can better adapt to mobile positioning in the experimental scene.

The proposed heat metering intelligent management platform will improve the heating satisfaction of residents. Through the intelligent management platform, the heating process will be fully and intelligently managed, and the heating customers will be provided with different heating by time, area, and temperature, and reasonable arrangements heat supply to improve the intelligence of heating users and greatly improve the quality of life of users.

The intelligent management of heat metering first realizes automatic metering, automatic statistical summary, and automatic billing of heat energy consumption; while reducing labor costs, it realizes real-time statistical summary. Second, the intelligent management of heat metering realizes the monitoring of the heating status and responds to accidents or failures in time. Realize customer relationship management for users again, understand user needs and feedback opinions in time, and realize online repairs and other functions. For decision-making, the intelligent management of heat metering adopts big data mining technology to analyze and predict the heating demand. Through the construction of the management information system, realtime interaction between the supply and demand parties is realized based on the Internet, which is convenient for users to inquire and understand heating consumption and tariffs. With the help of electronic payment methods such as online payment, heat metering energy management realizes all-round information management and services.

\section{Conclusion}

Heating in winter is an important livelihood project in northern my country, and it is also an issue that cannot be ignored in energy consumption and carbon emissions. Charging reasonable fees on the premise of ensuring people's livelihood has become an important topic of current heating management. Based on heating metering, Internet of Things, big data, cloud computing, and other technologies, intelligent management of heating production, distribution, metering, and billing is not only a problem of information and communication technology but also a new topic of efficient management automation and intelligence. The integration of management ideas and methods and artificial intelligence has given birth to the field of intelligent management. The intelligent management scheme of heating metering is only an attempt of intelligent management in the field of actual engineering, which has theoretical guiding significance and practical reference for other industries and fields. Combined with emerging technologies such as cloud computing and big data, the research in this article is not deep enough.

\section{Data Availability}

The data used to support the findings of this study are available from the corresponding author upon request.

\section{Conflicts of Interest}

The authors declare that they have no conflicts of interest.

\section{Acknowledgments}

This work was supported by Key Funding Project of Green Development Research Fund of Higher Education Ministry: Research on Intelligent Energy Conservation Strategy Based on Big Data (Educational Development 2016-07).

\section{References}

[1] S. Yi, "Research on the development mode of intelligent logistics based on internet of things and cloud computing in big data era," Revista de la Facultad de Ingenieria, vol. 32, no. 16, pp. 829-834, 2017.

[2] M. Babar and F. Arif, "Smart urban planning using big data analytics to contend with the interoperability in internet of things," Future Generation Computer Systems, vol. 77, pp. 65-76, 2017.

[3] S. Mohamed and S. Zulhuda, "Data protection challenges in the internet of things era: an assessment of protection offered by PDPA 2010," International Journal of Law Government and Communication, vol. 4, no. 17, pp. 1-12, 2019.

[4] E. D. Siew, R. K. Basu, H. Wunsch et al., "Optimizing administrative datasets to examine acute kidney injury in the era of big data: workgroup statement from the 15(th) ADQI consensus conference," Canadian Journal of Kidney Health and Disease, vol. 3, no. 1, p. 12, 2016.

[5] S. Akter and S. F. Wamba, "Big data analytics in e-commerce: a systematic review and agenda for future research," Electronic Markets, vol. 26, no. 2, pp. 173-194, 2016. 
[6] A. De Mauro, M. Greco, and M. Grimaldi, "A formal definition of big data based on its essential features," Library Review, vol. 65, no. 3, pp. 122-135, 2016.

[7] M. R. Palattella, M. Dohler, A. Grieco et al., "Internet of things in the 5G era: enablers, architecture, and business models," IEEE Journal on Selected Areas in Communications, vol. 34, no. 3, pp. 510-527, 2016.

[8] V. Pande, C. Marlecha, and S. Kayte, "A review-fog computing and its role in the internet of things," International Journal of Engineering Research and Applications, vol. 6, no. 10, pp. 2248-96227, 2016.

[9] A. V. Dastjerdi and R. Buyya, "Fog computing: helping the internet of things realize its potential," Computer, vol. 49, no. 8, pp. 112-116, 2016.

[10] H. Hamidi and M. Jahanshahifard, "The role of the internet of things in the improvement and expansion of business," Journal of Organizational and End User Computing, vol. 30, no. 3, pp. 24-44, 2018.

[11] C. Chang, S. N. Srirama, and R. Buyya, "Mobile cloud business process management system for the internet of things: a survey," ACM Computing Surveys, vol. 49, no. 4, pp. 1-42, 2016.

[12] G. Diega, "Clouds of things. data protection and consumer law at the intersection of cloud computing and the internet of things in the United Kingdom," Journal of Law \& Economic Regulation, vol. 9, no. 1, pp. 69-93, 2016.

[13] B. H. Dobkin, "A rehabilitation-internet-of-things in the home to augment motor skills and exercise training," Neurorehabilitation and Neural Repair, vol. 31, no. 3, pp. 217-227, 2017.

[14] H. Sun, X. Wang, R. Buyya, and J. Su, "CloudEyes: cloudbased malware detection with reversible sketch for resourceconstrained internet of things (IoT) devices," Software: Practice and Experience, vol. 47, no. 3, pp. 421-441, 2017.

[15] R. Visvanathan, D. C. Ranasinghe, A. Wilson et al., "Effectiveness of an ambient intelligent geriatric management system (AmbIGeM) to prevent falls in older people in hospitals: protocol for the AmbIGeM stepped wedge pragmatic trial," Injury Prevention Journal of the International Society for Child \& Adolescent Injury Prevention, vol. 25, no. 3, pp. 157-165, 2017.

[16] M. Zaharia, R. S. Xin, P. Wendell et al., "Apache spark: a unified engine for big data processing," Communications of the ACM, vol. 59, no. 11, pp. 56-65, 2016.

[17] Z. Obermeyer and E. J. Emanuel, "Predicting the future-big data, machine learning, and clinical medicine," New England Journal of Medicine, vol. 375, no. 13, pp. 1216-1219, 2016.

[18] D. Specht, "The data revolution. big data, open data, data infrastructures and their consequences," Media Culture \& Society, vol. 37, no. 7, pp. 1110-1111, 2016.

[19] J. Qin, H. Li, H. Li, X. Chen, and W. Feng, "Explore and practice of China's intelligent "new engineering"-based on the grounded theory," International Journal of Information and Education Technology, vol. 10, no. 8, pp. 632-640, 2020.

[20] C. Kolias, A. Stavrou, J. Voas, I. Bojanova, and R. Kuhn, "Learning internet-of-things security "hands-on"," IEEE Security \& Privacy, vol. 14, no. 1, pp. 37-46, 2016.

[21] J. Chen, Z. Lv, and H. Song, "Design of personnel big data management system based on blockchain," Future Generation Computer Systems, vol. 101, pp. 1122-1129, 2019.

[22] R. Parada, J. Melià-Seguí, and R. Pous, "Anomaly detection using RFID-based information management in an IOT context," Journal of Organizational and End User Computing, vol. 30, no. 3, pp. 1-23, 2018.
[23] M. Ali, L. T. Jung, A.-H. Abdel-Aty, M. Y. Abubakar, M. Elhoseny, and I. Ali, "Semantic-k-NN algorithm: an enhanced version of traditional k-NN algorithm," Expert Systems with Applications, vol. 151, p. 113374, 2020.

[24] Z. Lv, L. Qiao, J. Li et al., "Deep learning enabled security issues in the internet of things," IEEE Internet of Things Journal, vol. 8, no. 12, pp. 9531-9538, 2020. 